\title{
Numerical modeling and analysis of early shock wave interactions with a dense particle cloud
}

\author{
J. D. Regele, J. Rabinovitch, T. Colonius, and G. Blanquart ${ }^{\dagger}$ \\ California Institute of Technology, Pasadena, CA, 91125, U.S.A.
}

\begin{abstract}
Dense compressible multiphase flows exist in variable phase turbines, explosions, and fluidized beds, where the particle volume fraction is in the range $0.001<\alpha_{d}<0.5$. A simple model problem that can be used to study modeling issues related to these types of flows is a shock wave impacting a particle cloud. In order to characterize the initial shock-particle interactions when there is little particle movement, a two-dimensional (2-D) model problem is created where the particles are frozen in place. Qualitative comparison with experimental data indicates that the 2-D model captures the essential flow physics. Volume-averaging of the 2-D data is used to reduce the data to one dimension, and $x-t$ diagrams are used to characterize the flow behavior. An equivalent one-dimensional (1-D) model problem is developed for direct comparison with the 2-D model. While the 1-D model characterizes the overall steady-state flow behavior well, it fails to capture aspects of the unsteady behavior. As might be expected, it is found that neglecting the unclosed fluctuation terms inherent in the volume-averaged equations is not appropriate for dense gas-particle flows.
\end{abstract}

\section{Nomenclature}

$\alpha \quad$ Volume fraction

$\rho \quad$ Phase-averaged gas phase density

$p \quad$ Phase-averaged pressure

$u \quad$ Mass-averaged gas phase velocity

$e_{T} \quad$ Phase-averaged total energy

$h_{T} \quad$ Phase-averaged total enthalpy

$F_{D} \quad$ Drag force on a single particle

$n \quad$ Number density

$A_{p} \quad$ Particle Cross-sectional area

$D_{p} \quad$ Particle diameter

$x \quad$ Nondimensional position

$t \quad$ Nondimensional time

$V_{p} \quad$ Particle volume

Subscript

$c \quad$ Continuous phase

$d \quad$ Disperse phase

0 Reference state

$p \quad$ Particle

Superscript

Dimensioned quantity

*Postdoctoral scholar, Department of Mechanical Engineering; jonathan.regele@caltech.edu. AIAA Member.

†Assistant Professor, Department of Mechanical Engineering; g.blanquart@caltech.edu. AIAA Member. 


\section{Introduction}

Dense multiphase flows can be found in a variety of practical applications, including fluidized beds, variable phase turbines, and explosions. Variable phase turbines can be used in waste-heat recovery and geothermal power generation, but their efficiency is limited by the accuracy of modeling approaches used in the design phase. The ability to design a turbine accurately is dependent upon predicting mass, momentum, and energy transfer between phases as well as droplet formation, breakup, and collisions. Current state of the art models are often inadequate, and designers still rely heavily on experimental testing. Other fields, such as fluidized beds and explosives, suffer from similar modeling deficiencies.

Multiphase flow behavior can be divided into three main categories: dilute flow where the particle volume fraction is $\alpha_{d}<0.001$, collision-dominated flows where $0.001<\alpha_{d}<0.1$, and contact-dominated flows with $\alpha_{d}>0.1{ }^{1}$ Contrary to the former definition, the transition from collision dominated to contact dominated flow is defined differently in Ref. 2. They consider the transition to occur when the particle density is high enough that the disperse phase becomes a packed bed $\left(\alpha_{d}>0.5\right)$ and the flow can be considered granular. In dilute flows, collisions can essentially be neglected. ${ }^{1}$ In granular flows, particles are packed together such that particle motion is induced by direct inter-particle forces and gas propagation through the porous region between particles. Between these two limiting extremes lies gas-solid two-phase flow, where particle collisions and fluid drag both play major roles in flow behavior.

Drag coefficient correlations have been developed for dilute flows. ${ }^{3}$ However, for larger disperse phase volume fraction, the steady-state drag coefficient, $C_{D}$, deviates from the standard drag curve values. ${ }^{4,5,6}$ Consequently, voidage functions have been developed to modify the drag coefficient to account for this dependency, such that $C_{D}=C_{D 0} g\left(\alpha_{c}\right)$, where $g\left(\alpha_{c}\right)$ is the voidage function and $C_{D 0}$ is the drag coefficient of a single particle. ${ }^{4,5,7}$ However, these formulations have been developed for low Mach number flows where compressibility effects can be neglected. It is unclear how accurate they will be at capturing the drag coefficient in dense compressible multiphase flows.

A shock wave impacting a cloud of particles is a model problem that can be used to study shockparticle interactions and compressible multiphase flows. A combination of both experiments and numerical simulations have been used already to describe the response of a shock wave impacting dilute/dusty particle clouds . $8,9,10,11,12$

In the dilute regime, these flows are well characterized. In the granular regime where the particles are densely packed, continuum mixture theories exist to describe these flows. ${ }^{13,14}$ Baer $^{15}$ successfully modeled normal shock impingement experiments of Anderson, Graham, and Holman ${ }^{16}$ and Sommerfeld. ${ }^{17}$ Between these two limiting flow regimes, little information exists for gas-solid flows where the volume fraction is in the range $0.001<\alpha_{d}<0.5$. Boiko et al. ${ }^{18}$ performed experiments of shock waves impacting spatially inhomogeneous particle clouds in the range $0.001<\alpha_{d}<0.03$. In the case where $\alpha_{d}=0.03$, a reflected shock wave was observed, whereas in the more dilute cases no reflected shock was observed.

Recently, a multiphase shock tube experiment has been developed at Sandia National Labs. ${ }^{19,20}$ In this experiment, a shock wave $(M=1.67)$ impinges on a gravity fed particle cloud of width $L^{\prime}=3.2 \mathrm{~mm}$. The particle volume fraction inside the cloud is approximately $\alpha_{d}=0.15$ to 0.2 and the particles are of nearly uniform diameter. Detailed Schlieren images show the fluid response to the impact with the particle cloud. Reflected and transmitted shock waves are observed from the shock wave interaction with the cloud. For the first $30 \mu \mathrm{s}$, the cloud does not move, but after a few hundred microseconds, the trailing edge of the cloud travels several cloud lengths downstream, while the leading edge moves much less.

It is unclear what occurs when a shock wave impacts a dense particle field, particularly inside and just behind the particle cloud. The amount of information that can be obtained inside the cloud experimentally is limited. The goal of the present work is to perform a detailed investigation of the interaction between the incident shock wave and the particle cloud during the first several microseconds. Individual objectives of the current work are to a) develop an equivalent two-dimensional (2-D) model problem that captures the essential physics in this experiment, b) perform detailed 2-D numerical simulations of a shock wave impinging upon a cloud in order to characterize the flow behavior, c) compare the results with experimental data for qualitative consistency, d) perform one-dimensional (1-D) simulations using a standard volume fraction approach, and e) compare the two different model results and identify what details are captured and missing in the one-dimensional model. The present analysis is limited to the early "time" when the particles have not moved yet due to drag forces and can be assumed to be frozen in place.

The paper is organized as follows. First, a mathematical model is developed to describe the shock interaction behavior with a 2-D particle cloud. Then, results of the 2-D model and a discussion of the 
multidimensional effects and grid independence are presented in Section III. A 1-D volume-averaged model for direct comparison with the 2-D model is developed in Section IV. In Section V, the flow behavior is generalized, similarities and differences between the two models are highlighted, and additional terms that need to be modeled are identified. The paper concludes with a discussion of the current numerical results with the experimental data in Ref. 20.

\section{Mathematical Model}

The current work attempts to capture qualitatively the effects of a shock wave interacting with a cloud of spherical particles using a two-dimensional model. The Reynolds number based on the velocity behind the reflected shock and the particle diameter is approximately $R e=2000 .{ }^{20}$ Hence, the viscous forces on the particles are expected to be negligible, and the drag force on a particle is predominantly from drag.

The current work is focused on the initial transient interactions between the incident shock wave and the particle cloud. In the experiments in Ref. 20, this coincides with approximately the first 30-50 $\mu$ s. Experimental data during this time shows little, if any, particle movement, ${ }^{20,18,21}$ which makes it reasonable to assume that the particles are frozen in place. Furthermore, heat transfer can be neglected over such a short time frame. Finally, the particles are solid so there is no mass exchange between phases.

\section{II.A. Representation of particle cloud}

Clemins $^{22}$ demonstrated that to maintain small volume fraction fluctuations, $\langle\alpha\rangle-\hat{\alpha}$ (where $\langle\alpha\rangle$ is the volume average over a given sample volume and $\hat{\alpha}$ is the expected volume fraction), at least 60-150 particles are required inside of a 3-D sampling volume. Following his 3-D analysis, we estimate that between 15 and 30 particles should be sufficient in our 2-D simulations. The 2-D particle cloud is modeled using a staggered particle matrix with a total of 24 particles (approximately 5 particles in each direction). Figure 1 shows the arrangement of the particles for the two-dimensional simulations. By staggering each particle both in the $x$ - and $y$-directions with equal spacing, a relatively small number of particles can be used and still maintain small fluctuations, less than $\left\|\left\langle\alpha_{d}\right\rangle-\hat{\alpha}_{d}\right\|_{\infty}<0.008$. The sampling volume used to determine $\left\langle\alpha_{d}\right\rangle$ spans the entire cloud height in the $y$-direction, and a distance $D_{p} / 2$ in the horizontal direction, where $D_{p}$ is the particle diameter.

The particle diameter inside the cloud is related to the expected particle volume fraction, which is chosen to be $\hat{\alpha}_{d}=0.15$. In the 2-D model, the volume fraction is expressed as an area fraction by

$$
\hat{\alpha}_{d}=\frac{N \pi D_{p}^{2}}{4 L^{2}} .
$$

Here, $N$ is the total number of particles inside the square cloud of nondimensional length $L$ and area/volume fraction $\hat{\alpha}_{d}=0.15$. Since the length scale is nondimensionalized with respect to the cloud length $(L=1)$, the particle diameter is $D_{p}=0.089$ for $N=24$ particles. A full discussion of the nondimensionalization is presented in the next section.

The cloud is located between $x=-0.5$ and 0.5 , and the computational domain spans the range $x \in$ $[-2.5,3.5]$ and $y \in[-0.5,0.5]$. Inflow and outflow conditions are used on the left and right boundaries, respectively, and the top and bottom boundaries are periodic to simulate an infinitely tall cloud. The simulation uses a cartesian grid. The grid spacing is uniform in the $y$-direction and in the $x$-direction for $x \in[-1.5,1.5]$. In this region, the cells have unity aspect ratio, $\Delta x=\Delta y$. Outside of this range, unequal spacing is used in the $x$-direction.

Simulations were performed for four different levels of resolution to determine grid sensitivity. The number of cells used to resolve the particles in the four different cases were 13, 26, 52, and 105 cells per particle.

It should be reiterated that the two-dimensional model described above is not intended to provide a quantitatively accurate comparison with experimental data. ${ }^{20}$ The current motivation is to capture qualitatively the essential physics in two dimensions and use the volume-averaged solution to determine what the 1-D model is capable of capturing and what needs to be modeled. Full three-dimensional simulations for quantitative comparison with experimental data will be the subject of future work. 


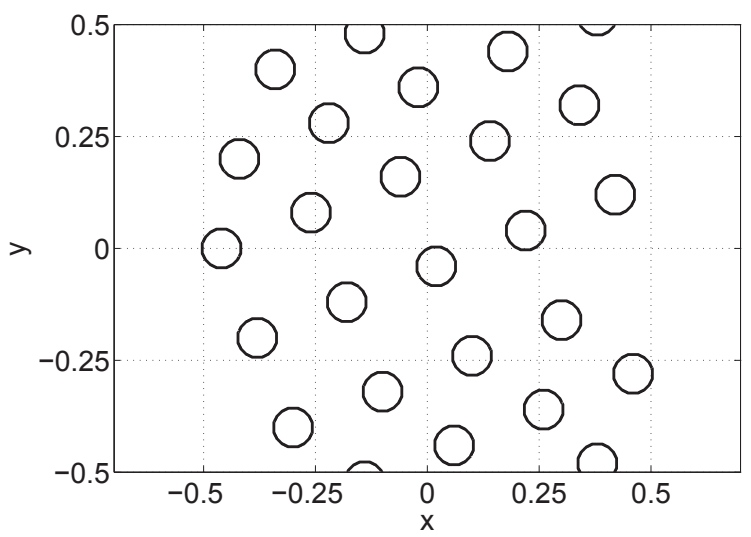

Figure 1. An array of particles is arranged in such a way that the phase-averaged volume fraction deviates from the prescribed continuous phase volume fraction $\alpha_{c}=0.85$ by less than $0.8 \%$.

\section{II.B. 2-D Equations}

The two-dimensional computations are conducted using the Euler equations. The particles are modeled as solid cylinder walls and discretized using a stair-step approach. Slip wall conditions are imposed on the cylinder walls in addition to the zero penetration condition. The nondimensional Euler equations are given by

$$
\begin{aligned}
\frac{\partial}{\partial t} \rho+\frac{\partial}{\partial x_{i}} \rho u_{i} & =0 \\
\frac{\partial}{\partial t} \rho u_{i}+\frac{\partial}{\partial x_{j}} \rho u_{i} u_{j}+\frac{\partial}{\partial x_{i}} p & =0 \\
\frac{\partial}{\partial t} \rho e_{T}+\frac{\partial}{\partial x_{i}}\left[u_{i}\left(\rho e_{T}+p\right)\right] & =0,
\end{aligned}
$$

where the non-dimensional quantities are are defined with respect to the initial undisturbed dimensional gas state $\left[a_{0}^{\prime}, \rho_{0}^{\prime}\right]$ and a dimensional length scale $L^{\prime}$ defined as the thickness of the cloud. Primes are used to denote dimensional quantities. With this formulation, the non-dimensional quantities are expressed in terms of dimensional quantities as $x=x^{\prime} / L^{\prime}, t=t^{\prime} a_{0}^{\prime} / L^{\prime}, u=u^{\prime} / a_{0}^{\prime}, \rho=\rho^{\prime} / \rho_{0}^{\prime}, p=p^{\prime} /\left(\rho_{0}^{\prime} a_{0}^{\prime 2}\right)$ and $e_{T}=e_{T}^{\prime} / a_{0}^{\prime 2}$.

The equation of state for a perfect gas is

$$
p=(\gamma-1) \rho e=(\gamma-1)\left(\rho e_{T}-\frac{1}{2} \rho u_{i} u_{i}\right),
$$

where $e$ is the internal energy, $e_{T}$ is the total energy, and $\gamma$ is the specific heat ratio.

\section{II.C. Numerical Method}

The 2-D simulations are performed using the NGA code. The Roe-Pike method ${ }^{23}$ combined with MUSCLHancock data reconstruction ${ }^{24}$ is used to solve the Euler equations (Eq. 2). The van Leer limiter ${ }^{25}$ is used to minimize the effects of artificial viscosity/diffusion while maintaining a total variation diminishing

solution. The method is first order accurate near shocks and contact discontinuities, and second order accurate elsewhere.

\section{II.D. Initial Conditions}

The initial nondimensional Rankine-Hugoniot shock jump conditions for the $M=1.67$ shock wave are

$$
\begin{array}{rr}
p_{5}=2.21 & p_{0}=0.714 \\
\rho_{5}=2.15 & \rho_{0}=1 \\
u_{5}=0.896 & u_{0}=0
\end{array}
$$

where subscript 5 denotes the fluid condition upstream of the incoming shock and subscript 0 indicates the undisturbed fluid downstream of the shock. The reason for this notation will be apparent shortly. 


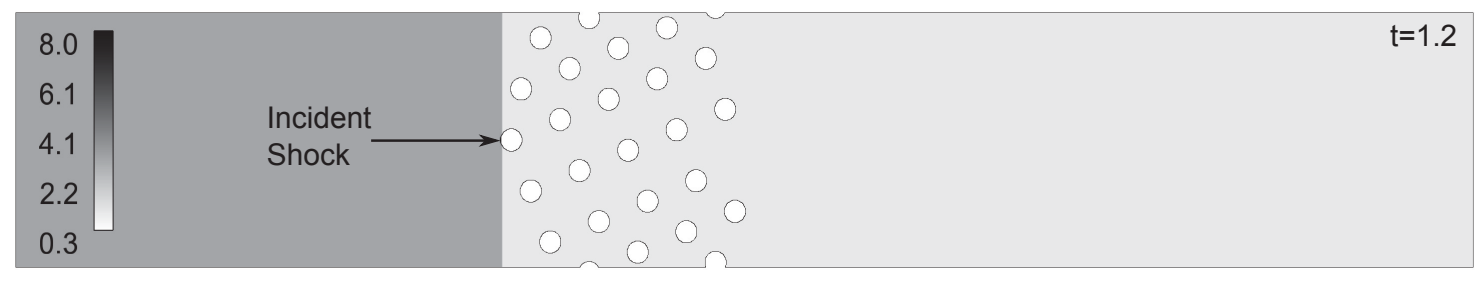

(a)
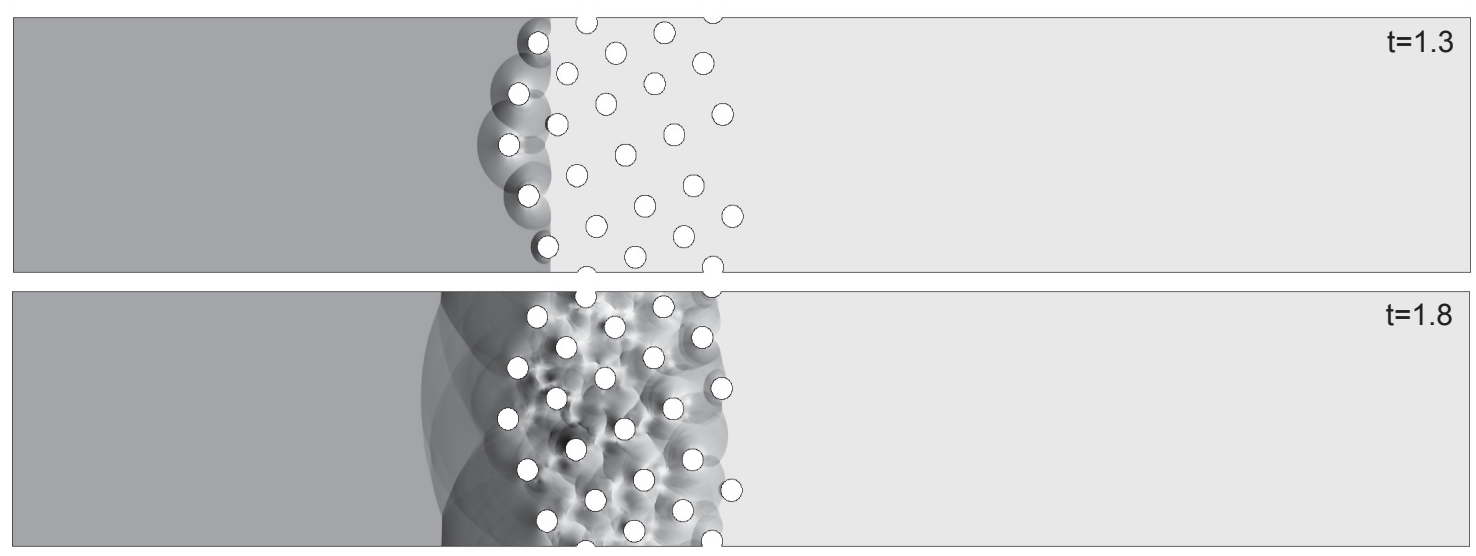

(c)
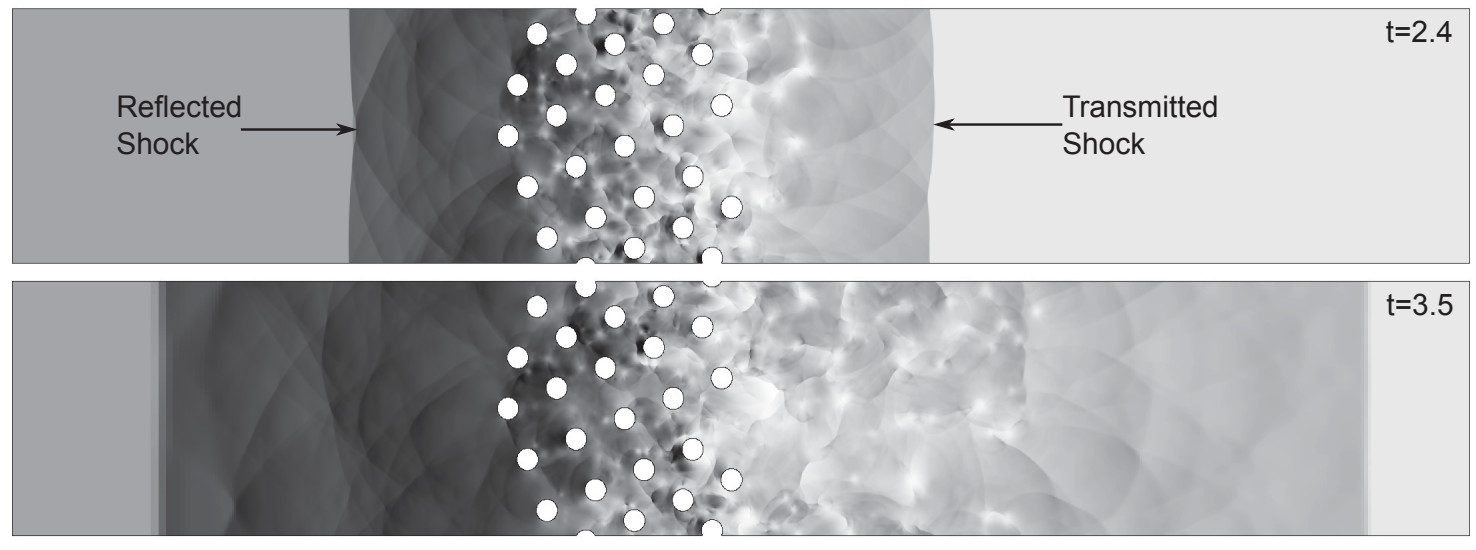

(d)

(e)

Figure 2. A time series evolution of the normalized pressure $\left(p / p_{0}\right)$ shows the reflected and transmitted shock waves, as well as unsteady flow conditions both inside and behind the cloud.

\section{2-D Results Overview}

Much of the results can be described with a 1-D description, yet the results clearly show multidimensional effects. This section will focus on the effects of multiple dimensions and grid independence.

\section{III.A. General flow features}

Figure 2 shows a time series evolution of two-dimensional normalized pressure $\left(p / p_{0}\right)$ contours at several different points in time. When the incident shock first hits the particle cloud, a reflected shock wave is created (Fig. 2b). The reflected shock wave is comprised of multiple different shock reflections, one from each particle, which cumulatively interact to form a nearly planar shock front. The initial reflected shock wave has a relatively small magnitude (Fig. 2c) in comparison to later times (Fig. 2d). As time proceeds, additional reflected waves from other particles within the cloud contribute to strengthening the amplitude of the reflected shock (Fig. 2e). Figure 2 also shows a transmitted shock that traverses through the cloud and into the undisturbed gas downstream.

The interaction of the shock wave with each cylinder in the current work is very similar to that characterized by Sun et al. ${ }^{26}$ When the incident shock wave impacts the cylinder, a regular reflection is formed. As the incident shock travels further along the cylinder, the regular reflection transitions to a Mach reflection. When the Mach reflections converge on the line of symmetry at the trailing edge of the cylinder, a region of 


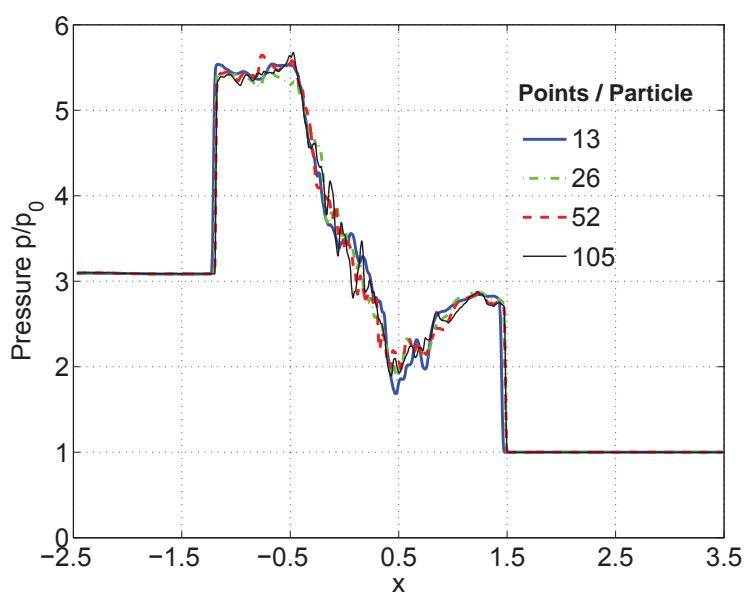

Figure 3. The number of points required to resolve a single particle was varied by almost an order of magnitude. These phase-averaged pressure profiles at $t=2.5$ indicate that the solution is independent of grid resolution.

high pressure is created, which subsequently expands and the pressure decreases.

Flow separation at the trailing edge of the cylinder is not observed until the transmitted shock wave has traveled approximately one diameter downstream of the cylinder. Shortly thereafter (about 0.01 time units), a recirculation zone forms behind the cylinder. The recirculation zone is initially symmetric, after which unsteady vortex shedding commences. Although there is no physical viscosity present in the Euler equations, numerical viscosity is implicitly present. This numerical viscosity causes the initial flow separation to occur, and is discussed in detail in the next section.

The pressure behind the transmitted shock that initially emerges from the cloud has an average magnitude of $p / p_{0}=2.4$, which is $20 \%$ lower than that of the initial shock. This trend is consistent with the experimental observations. The pressure drop is due to the multiple shock reflections created from each particle interaction as the shock traverses the cloud. After the transmitted shock emerges from the cloud, the pressure at the trailing edge of the cloud continues to drop as unsteady wakes form behind each cylinder, which increases the drag and thereby further reduces the pressure.

Between the transmitted shock wave and the trailing edge of the cloud lies a region of fluid that is strongly unsteady and consists of vortices shed by each particle. Inside that region, localized pockets of low pressure exist in the vortex cores. This pressure may be as low as $p / p_{0}=0.3$, which is lower than the initial undisturbed gas condition. A quantified discussion of this region will be presented in Section V.

\section{III.B. Grid Dependence}

As a result of the large particle Reynolds number found in the experiment (near $R e=2000$ ), the Euler equations have been used as a first approach to perform the 2-D numerical computations. The numerical method implicitly contains numerical viscosity in order to capture shocks and contact discontinuities.

The drag coefficient for Reynolds numbers in the range $10^{3}<R e<10^{5}$, as found in the experiment, are relatively independent of Reynolds number. ${ }^{27}$ Since the numerical method implicitly contains numerical viscosity, a grid dependence study was performed to determine how sensitive the solution is to the numerical viscosity. It is not possible to obtain point-wise convergence using the Euler equations. Thus, to assess grid sensitivity, planar-average quantities are used to gauge solution dependence.

2 -D simulations were performed using $13,26,52$, and 105 points per cylinder diameter. There is little noticeable difference in the mean values between the four different levels of resolution. Therefore, it is reasonable to compare the 2-D results with the 1-D planar average model. While the numerical viscosity is not equivalent to molecular viscosity, the particle boundary layer wakes contain many features expected in a real flow (albeit three-dimensional).

\section{Phase Averages and 1-D Model}

In this section, a 1-D model is developed to simulate the planar-averaged flow. Volume averaging procedures are defined and used to formulate the 1-D model equations. Discussion of the drag coefficient required 
to close the 1-D model is provided.

\section{IV.A. Definition of Averages}

First, the volume average definitions must be made. The volume average is defined

$$
\bar{\phi}=\frac{1}{V} \int_{V} \phi d V,
$$

where $V$ is the sampling volume including both the continuous and disperse phases. If the volume integral is limited to the continuous phase, the phase average (or Reynolds average) is defined

$$
\langle\phi\rangle=\frac{1}{V_{c}} \int_{V_{c}} \phi d V,
$$

where $V_{c}$ is the volume inside $V$ that only includes the continuous phase, so that the Reynolds and volume averages are related by

$$
\bar{\phi}=\alpha_{c}\langle\phi\rangle \text {. }
$$

Finally, the mass average, or Favre average is defined as

$$
\widetilde{\phi}=\frac{\overline{\rho \phi}}{\bar{\rho}}=\frac{\langle\rho \phi\rangle}{\langle\rho\rangle} .
$$

Volume-averages in the transverse direction over the continuous phase are used to obtain one-dimensional planar averaged quantities from the two-dimensional solutions for comparison with the 1-D model. The phase-averaged quantity $\langle B\rangle(x)$ for a quantity $B(x, y)$ is obtained using Eq. 6, where $V_{c}$ is a sampling volume that is thin in the $x$-direction and spans the entire domain height in the $y$-direction. This equation is used to determine the phase-averaged quantities for the conserved variables $\rho, \rho u_{i}$, and $\rho e_{T}$ at each $x$-position on the numerical grid. The primitive variables $u_{i}$ and $p$ are determined from these quantities.

\section{IV.B. One-dimensional Equations}

The one-dimensional volume-averaged model in this work is based upon the averaging procedures for twophase gas-particle flows presented in Crowe et al. ${ }^{1}$ The Navier-Stokes equations form the foundation of the model. Due to the short timescales under consideration, the interphase mass and heat transfer are considered negligible. The main effects of viscous stresses are neglected except through their impact on the drag law.

As for the 2-D simulations, the particles are frozen in place, such that the particle velocity is zero and the volume fraction is only a function of position $\alpha=\alpha(x)$. Momentum coupling between the two phases is assumed to have dominant contributions from the undisturbed flow force ${ }^{1}$ and the steady-state drag law. With these assumptions, the one-dimensional, continuous phase, volume-averaged conservation equations are

$$
\begin{aligned}
\frac{\partial}{\partial t}\left(\alpha_{c}\left\langle\rho_{c}\right\rangle\right)+\frac{\partial}{\partial x}\left(\alpha_{c}\langle\rho\rangle \tilde{u}\right) & =0 \\
\frac{\partial}{\partial t}\left(\alpha_{c}\left\langle\rho_{c}\right\rangle \tilde{u}\right)+\frac{\partial}{\partial x}\left(\alpha_{c}\left\langle\rho_{c}\right\rangle \tilde{u}^{2}+\langle p\rangle\right) & =\alpha_{d} \frac{\partial\langle p\rangle}{\partial x} \\
& -\frac{\alpha_{d}}{2} \frac{A_{p}}{V_{p}} C_{D}\left\langle\rho_{c}\right\rangle|\tilde{u}| \tilde{u} \\
\frac{\partial}{\partial t}\left(\alpha_{c}\left\langle\rho_{c}\right\rangle \tilde{e}_{T}\right)+\frac{\partial}{\partial x}\left(\alpha_{c}\left\langle\rho_{c}\right\rangle \tilde{u} \tilde{h}_{T}\right) & =0
\end{aligned}
$$

where the unclosed fluctuation terms are neglected. The total energy and enthalpy are defined by

$$
\begin{aligned}
\langle\rho\rangle \tilde{e}_{T} & =\left\langle\rho_{c}\right\rangle \tilde{e}+\frac{1}{2}\left\langle\rho_{c}\right\rangle \tilde{u}^{2}, \\
\left\langle\rho_{c}\right\rangle \tilde{h}_{T} & =\left\langle\rho_{c}\right\rangle \tilde{e}+\frac{1}{2}\left\langle\rho_{c}\right\rangle \tilde{u}^{2}+\langle p\rangle,
\end{aligned}
$$




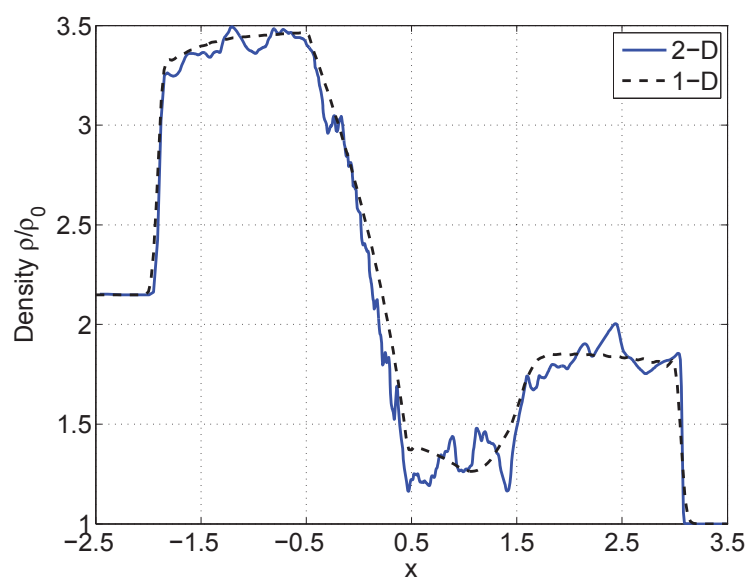

(a) Density

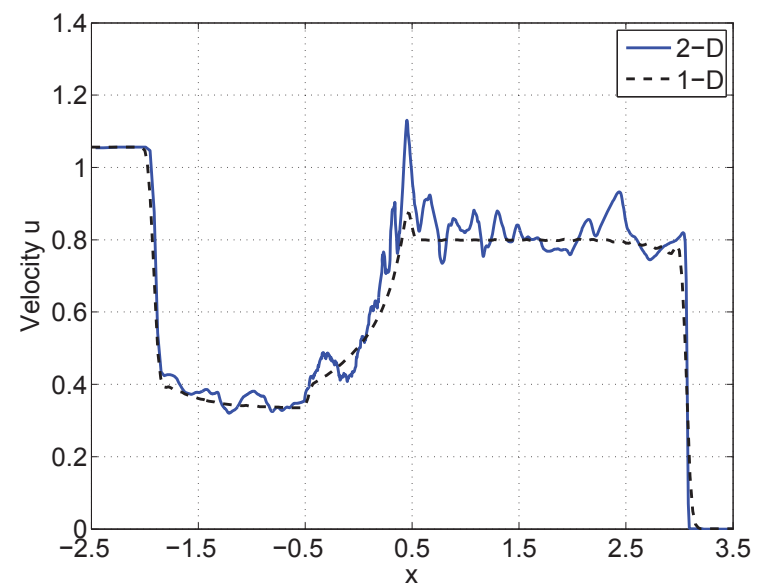

(b) Velocity

Figure 4. A comparison between the 1-D and 2-D phase-averaged solutions at $t=3.5$ indicate that the density and $x$-velocity of the 2-D phase-averaged solution oscillates around the one-dimensional model solution.

where $\tilde{e}$ is the internal energy. In Eq. $9, V_{p}$ is the volume of a single particle, $\alpha_{c}$ is the continuous phase volume fraction, $\alpha_{d}=1-\alpha_{c}$ is the disperse phase volume fraction, $C_{D}$ is the drag coefficient for a single particle, and $A_{p}$ is the particle cross-sectional area.

The drag coefficient $C_{D}$ is a function of the particle shape, Reynolds number $R e$, Mach number $M$, volume fraction $\alpha_{c}$, and the surface roughness. Since the 2-D model uses the Euler equations, the theoretical Reynolds number is infinite. However, it was demonstrated in Section III.B that the 2-D solutions are independent of $R e$, which eliminates one of drag coefficient parameters. The drag coefficient $C_{D}$ is determined with the 1-D model by finding the value that matches best the reflected and transmitted shock locations and magnitudes.

The cross-section to volume ratio $A_{p} / V_{p}$ for a cylinder is

$$
\frac{A_{p}}{V_{p}}=\frac{4}{\pi D_{p}},
$$

where $D_{p}$ is the non-dimensional cylinder diameter.

Equations 9 are solved using the compressible flow solver developed for the dynamically Adaptive WaveletCollocation Method (AWCM). ${ }^{28}$

\section{IV.C. One-Dimensional Domain}

In the one-dimensional approach, the volume-averaged equations are used to describe the presence of particles. The continuous phase volume fraction $\alpha_{c}$ in the one-dimensional model is constructed to reflect accurately the phase-average volume fraction in the 2-D model. The computational domain is in the range $x \in[-4,6]$ with a total of 400 points across the domain. The initial flow conditions are identical to the 2-D model defined in Section II.D.

To assess the sensitivity of the results to the grid resolution, the grid was increased by up to 4 times with a total of 1600 points. The solution results remained unaffected, with the exception of the reflected and transmitted shock thicknesses. This is expected because the numerical scheme is first order accurate at these locations.

\section{IV.D. Drag Coefficient}

The drag coefficient that most accurately captures the transmitted and reflected shock wave positions was found to be $C_{D}=3.3$. Figure 4 compares the average density and velocity profiles for both the 2-D and 1-D model results at $t=3.5$. It is interesting to note that both the reflected and transmitted shock positions are nearly identical, between the 1-D and 2-D results. Additionally, the 2-D results appear to oscillate around the 1-D model results for a significant portion of the solution. This indicates that the steady-state drag law is relatively accurate in capturing the basic flow phenomena captured in the 2-D model. 


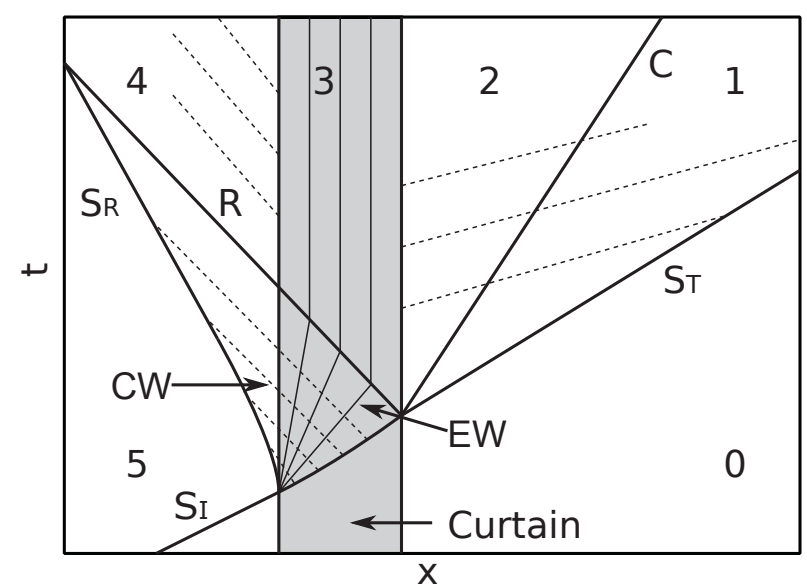

Figure 5. An $x$ - $t$ diagram demonstrates the one-dimensional behavior observed in both the one- and two-dimensional model results. Six different regions are identified, which include the (0) undisturbed gas, (1) fluid behind the transmitted shock $S_{T}$, (2) fluid between the contact $C$ and the cloud's trailing edge, (3) expansion across the cloud, (4) fluid behind the reflected shock, and (5) incident shock condition.

The drag coefficient $C_{D}=3.3$ is a factor of 2.8 higher than the expected single cylinder value $C_{D} \approx 1.2$ for an incompressible flow at $R e=2000$. This increase in drag coefficient has two main origins: the close packing of particles and compressibility effects.

In incompressible dense particle clouds, the drag coefficient on a single particle is increased due to the close proximity of neighboring particles. As discussed earlier, numerous different models have been proposed that modify the drag coefficient to account for a large disperse phase volume fraction. ${ }^{4,5,7,29}$ Each of these models involve a correction to the drag coefficient or friction factor in such a way that the drag force in a particle cloud can be expressed as $F_{D}=g\left(\alpha_{c}\right) F_{D_{0}}$, where $F_{D_{0}}$ is the drag force using the standard drag coefficient, and $g\left(\alpha_{c}\right)$ is a correction function (voidage function ${ }^{7}$ ).

In addition to close particle proximity, the drag in the current model is anticipated to depend on the flow Mach number as well. Ideally, one could formulate a correlation of the form

$$
F_{D}=g\left(\alpha_{c}\right) h(M) F_{D 0}
$$

where the particle proximity and Mach number effects are independent. However, the presence of transonic flow conditions and unsteady compression wave generation from the cloud suggests that the effects of these two parameters are likely to be highly linked in the present 2-D simulations.

If Eq. 13 were assumed to be true and that $g\left(\alpha_{c}\right)$ for spheres resembles $g\left(\alpha_{c}\right)$ for cylinders, the increase in drag coefficient can be estimated. The model of di Felice predicts $g\left(\alpha_{c}\right)=1.8$ for $R e=2000$ and $\alpha_{c}=0.85$. The average Mach number across the cloud is $M \approx 0.4$. With this Mach number, the drag coefficient is expected to increase by a factor of $1.1 .^{27}$ If this were the case, the product of $g\left(\alpha_{c}\right)=1.8$ and $h(M)=1.1$ gives an increase of 2.0 (instead of 2.8), which is roughly $30 \%$ less.

\section{Comparison of 1-D and 2-D Models}

\section{V.A. General Flow Description}

The general behavior of the shock interaction with the particle cloud is best described using an $x$ - $t$ diagram. Figure 5 shows a typical $x$ - $t$ diagram that is specific to the early interactions where the particles can be assumed to be frozen in place. Similar $x$ - $t$ diagrams can be found in Rogue et al. ${ }^{6}$ and Miura and Glass. ${ }^{10}$ Figure 5 shows six different fluid regions, which include the (0) undisturbed gas, (1) fluid behind the transmitted shock $\mathrm{S}_{T}$, (2) fluid between the contact $\mathrm{C}$ and the cloud's trailing edge, (3) the expansion across the cloud, (4) the fluid behind the reflected shock $\mathrm{S}_{R}$, and (5) flow conditions upstream of the initial shock $\mathrm{S}_{I}$.

When the original shock impacts the leading edge of the cloud, a portion of it is transmitted $\left(\mathrm{S}_{T}\right)$ and part of it is reflected $\left(\mathrm{S}_{R}\right)$. An expansion fan moves through the cloud behind the transmitted shock $\mathrm{S}_{T}$, which starts the formation of the pressure gradient across the cloud. When the transmitted shock emerges from the cloud's trailing edge, a contact discontinuity $\mathrm{C}$ and a rarefaction wave $\mathrm{R}$ are created. The contact propagates downstream and separates region 1 and 2. The rarefaction wave stops the expansion fan's propagation and 


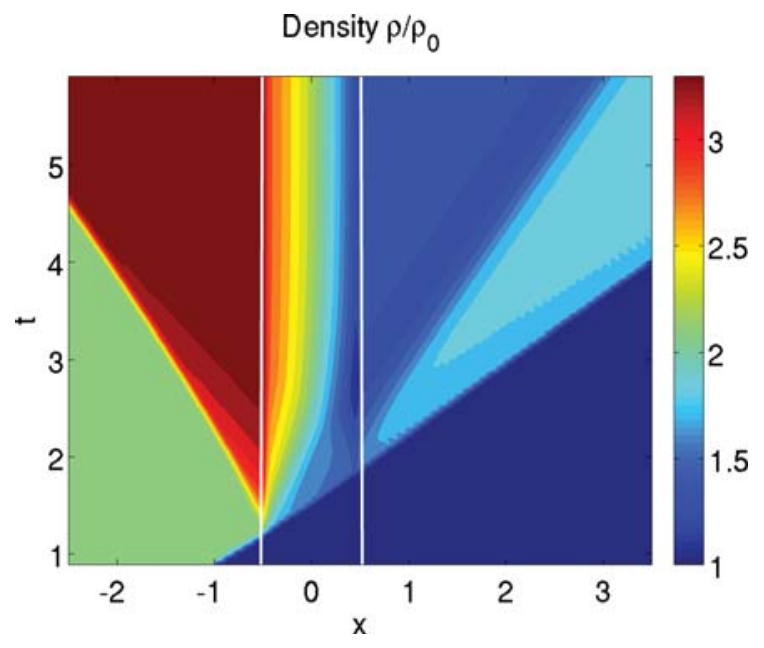

(a) One-dimensional density

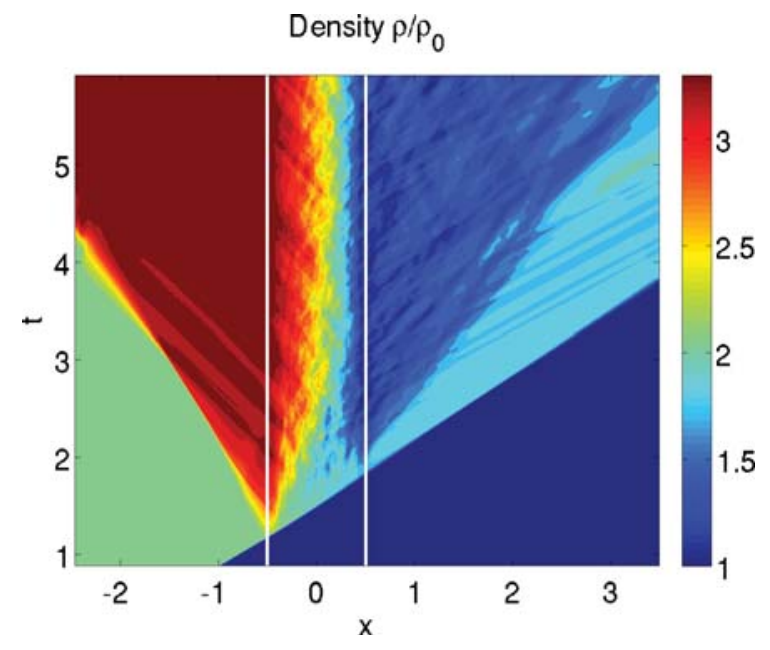

(b) Two-dimensional density

Figure 6. $x-t$ diagrams for density compare the (a) one-dimensional and (b) two-dimensional planar average solutions.

sets up a steady-state pressure gradient across the cloud (region 3). Additionally, when the rarefaction reaches the leading edge of the cloud, the pressure in region 4, which was gradually increasing from the cumulative addition of compression waves CW, becomes constant.

The steady-state pressure gradient will not form immediately after the rarefaction $\mathrm{R}$ passes back through the cloud. Instead, the pressure inside the cloud will fluctuate about its mean value, emitting acoustic waves away from the cloud. These fluctuations are shown in Fig. 5 as dashed lines propagating away from the cloud (labeled CW) along the characteristic lines $u-c$ and $u+c$.

\section{V.B. Model Similarities}

As mentioned earlier, one-dimensional quantities can be obtained from the 2-D data by taking the phaseaverage defined in Eq. 6. The 2-D solution used for comparison is the case where 52 grid cells are used per cylinder diameter. The sampling volume spans $D_{p} / 2$ in the $x$-direction and the entire domain in the $y$-direction. In order to prevent shock widening from the volume averaging process, sampling volumes of a single grid cell in the $x$-direction are used in regions where the reflected and transmitted shocks are located.

Figures 6,7 , and 8 compare $x$ - $t$ diagrams for the density, velocity, and pressure, respectively. Both the 1-D and 2-D models show the basic flow behavior described in the $x$ - $t$ diagram (5) of the previous section. In the density diagram (Fig. 6), both models show the reflected $\left(\mathrm{S}_{R}\right)$ and transmitted $\left(\mathrm{S}_{T}\right)$ shocks when the incident shock $\mathrm{S}_{I}$ first impacts the particle cloud. The expansion across the cloud is captured both during its formation and after it has formed a steady-state expansion. The existence of the contact discontinuity $\mathrm{C}$ is consistent between both models, and the density magnitude and slope are similar as well.

The velocity in Fig. 7 is similar between both models. Although the velocity contour shows some unsteadiness primarily behind the cloud, Fig. 4(b) indicates that at $t=3.5$ the 2 -D solution oscillates about the 1-D solution velocity.

\section{V.C. Model Differences}

There is a noticeable difference in behavior during the initial transient period $(1.2<t<1.8)$ from when the shock wave first enters the cloud until it leaves. The transmitted density is higher in the 2-D model (Fig. 6(b)) than it is in the 1-D model (Fig. 6(a)). This is attributed to the fact that as the shock first passes through the cloud, the flow around the cylinders has not detached yet to form a wake. The drag coefficient in the steady-state model assumes a fully developed and separated wake, which causes the 1-D model to overestimate the particle drag during the initial shock propagation.

Figure 7 shows that the shock traverses the cloud with a higher speed in the 2-D model than it does in the 1-D model. For each point in space inside the cloud just after the shock has passed, the velocity remains high for $t \approx 0.2$ and then the mean velocity drops rapidly. The rapid drop in velocity is characterized by 


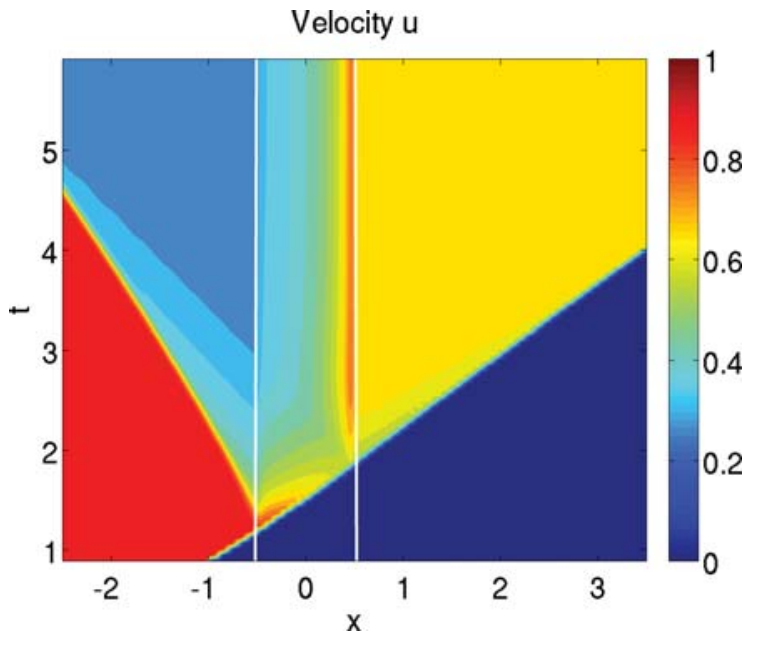

(a) One-dimensional velocity

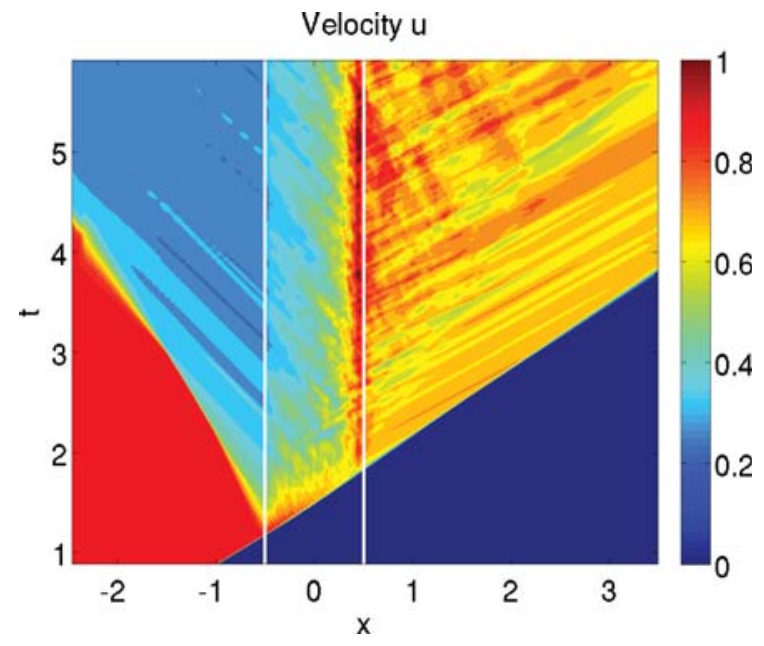

(b) Two-dimensional velocity

Figure 7. $x$ - $t$ diagrams for velocity compare the (a) one-dimensional and (b) two-dimensional planar average solutions.

the onset of flow separation and wake formation. In the 1-D model, the velocity experiences a more gradual decay and this dynamic process is not captured.

In Fig. 8, the 2-D $x$ - $t$ diagram shows the presence of coherent compression waves propagating in both the $-x$ and $+x$-directions away from the cloud region. These waves are absent in the 1-D model. Inside the cloud of the 2-D model, it is difficult to trace the characteristic lines, which suggests that the compression waves originate from the cloud itself. This is consistent with the observation of coherent localized compression waves in the 2-D pressure contour plot in Fig. 2. These waves appear to be repeated internal reflection/transmission waves created by the initial shock that continue to reverberate inside the cloud.

Pressure is normally constant across a true contact discontinuity. In the 1-D model, this is shown to be true (see Fig. 8(a)). In the 2-D model, the contact separates a region of predominantly steady flow (only disturbed by weak periodic acoustic waves), and a region of unsteady flow with large oscillating pressure disturbances associated with vortex shedding.

Figure 9 compares the pressure at $t=3.5$ for the 1 -D and 2-D models. The average pressure between the cloud trailing edge at $x=0.5$ and the contact discontinuity at $x=1.5$ is consistently lower than that predicted by the 1-D model. In its current form, the 1-D model is incapable of reproducing this unsteady behavior. Since the unsteady flow features travel downstream at the mean fluid velocity, the contact discontinuity marks the transition between a smooth transmitted shock region and an unsteady wake region.

The equations solved in the 1-D model do not include the unclosed fluctuation terms created during the volume-averaging procedure. ${ }^{1}$ This is a reasonable assumption in dilute multiphase flows, such as dusty gases. ${ }^{9}, 10,11,12,30$ However, in dense flows this assumption may not be appropriate. The volume-averaged momentum equation (Eq. 9b) contains one unclosed Reynolds stress term. ${ }^{1,31}$ It is convenient to define a total pressure $p_{T}$ to be the sum of the volume-averaged pressure $\langle p\rangle$ and the Reynolds stress

$$
p_{T}=\langle p\rangle+\alpha_{c}\left\langle\rho_{c} u^{\prime \prime} u^{\prime \prime}\right\rangle .
$$

This is the 1-D equivalent formulation of the multidimensional pressure tensor. ${ }^{1,31}$

Figure 9 shows the 1-D and 2-D pressures $\langle p\rangle$, as well as the the total pressure $p_{T}$. The addition of the unclosed fluctuation Reynolds stress term accounts for the difference between the solutions. The 2-D $\langle p\rangle$ and $p_{T}$ quantities are identical outside of the cloud and the unsteady fluctuation region, but only the total pressure matches accurately the 1-D model solution inside the cloud and unsteady region. In order to model these interactions accurately using a 1-D model, models must be created that capture these unsteady effects. 


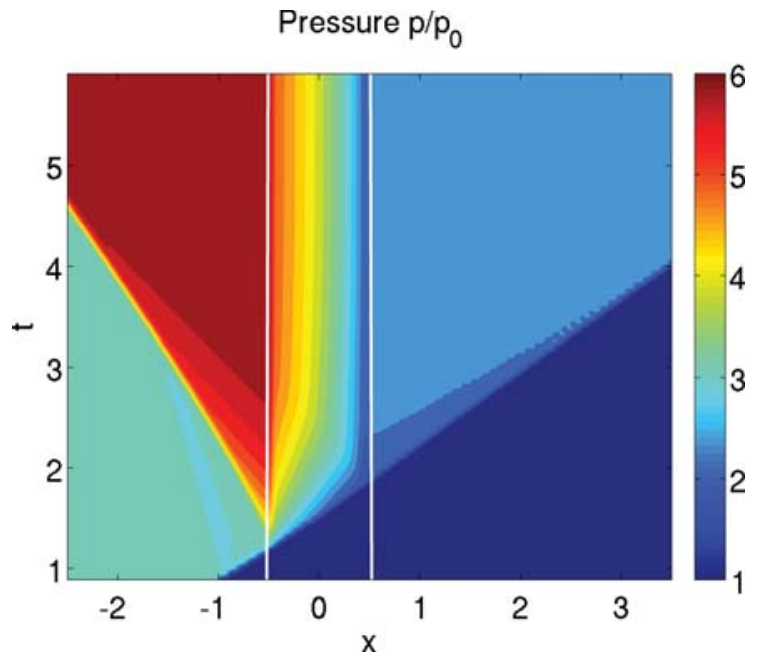

(a) One-dimensional pressure

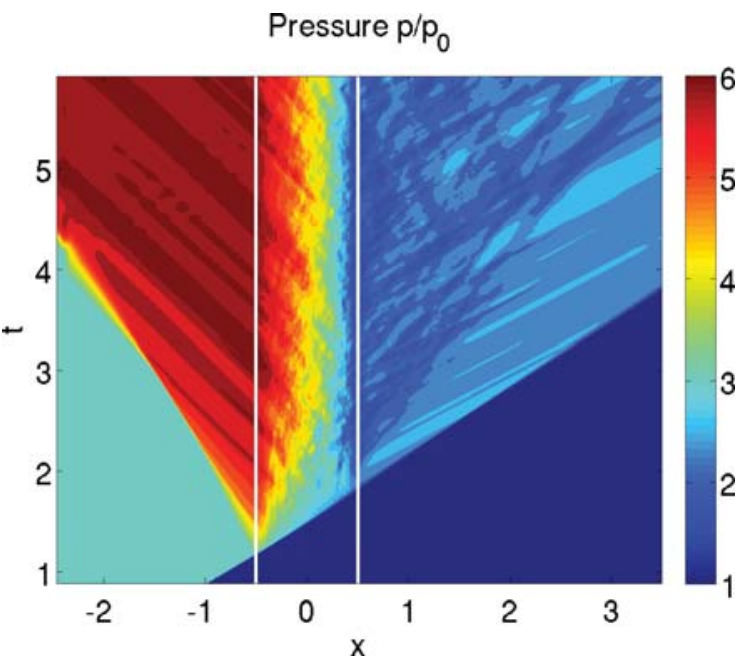

(b) Two-dimensional pressure

Figure 8. $x-t$ diagrams for pressure compare the (a) one-dimensional and (b) two-dimensional planar average solutions. The contact discontinuity at $x=1.5$ marks the interface between steady and unsteady flow regions.

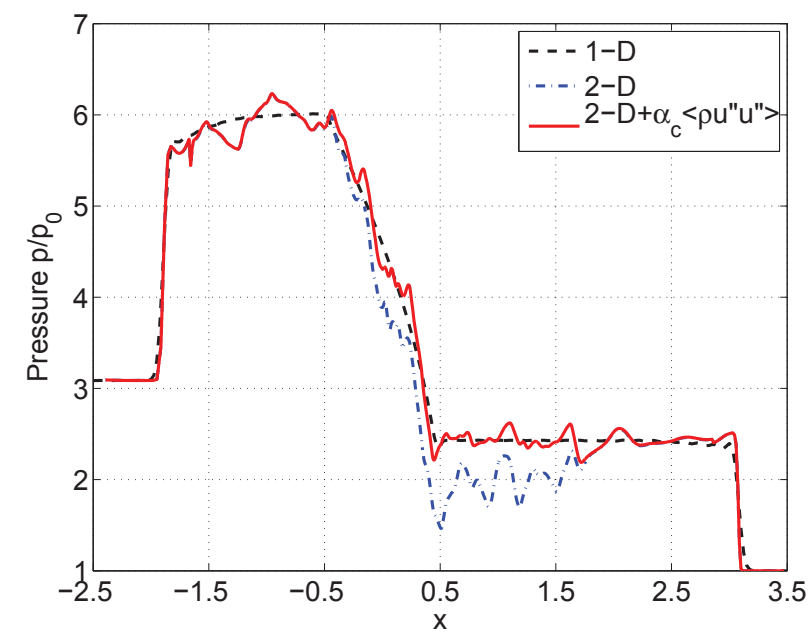

Figure 9. At $t=3.5$, the 2-D planar averaged solution is lower than the 1-D model between the cloud's trailing edge and the contact discontinuity.

\section{Discussion}

It is difficult to make a direct comparison of the current 2-D work with that observed experimentally in Ref. 20 for two primary reasons: 1) because of the difference in dimensionality (2-D vs. 3-D), and 2) the timescales of interest in the experiment are over several hundred microseconds, whereas the timescales of interest in this work are only over the first $30 \mu \mathrm{s}$. However, further insight into the fine details of the flow behavior can still be made.

In the experiment, ${ }^{20}$ the momentum and energy fluxes downstream of the cloud are reduced by $30-40 \%$, which is a substantially larger decrease than that observed in dusty gases. ${ }^{10}$ The momentum and energy flux changes predicted in the current work show a similar large reduction (about 45\%). This larger value is expected because of the higher particle drag on cylinders compared to spheres.

In Ref. 20, the Schlieren images show transmitted and reflected shock waves. Behind the transmitted shock is a region of relatively constant density fluid. Between the transmitted shock and the trailing edge of the cloud, there is a region of darkness closer to the cloud's trailing edge and a light colored region closer to the transmitted shock. Based upon the current analysis, it seems that this transition from light to dark may actually be the contact discontinuity, where the light color in the Schlieren image depicts the drop in density across the contact. The presence of this contact is consistent with that in Ref. 10. The unsteady 
region between the contact and the cloud's trailing edge may appear dark in the Schlieren images ${ }^{20}$ because the image is created with a plane-averaged line of sight.

From this perspective, the 2-D model seems to capture the essential flow physics. The transmitted and reflected shock waves regain a planar shape after emerging from the cloud. A contact discontinuity is formed at the trailing edge of the cloud and marks the interface between the laminar fluid in region 1 and an unsteady region 2. The flow in region 2 may be turbulent, but this cannot be confirmed without performing three-dimensional computations.

\section{Conclusions}

One- and two-dimensional simulations have been performed to study the early stages after a shock wave impinges normally upon a planar cloud of particles. Two-dimensional simulations are performed using a finite number of particles, which are modeled as cylinders frozen in space. Planar phase-averages are used to create equivalent one-dimensional profiles.

The $x$ - $t$ diagrams show reflected and transmitted shock waves. An expansion fan propagates into the cloud and is stabilized in place by the passage of a rarefaction wave that is created after the transmitted shock emerges from the cloud. This creates a steady-state expansion across the particle cloud that accelerates the flow. Finally, a contact discontinuity is formed at the cloud's trailing edge when the transmitted shock emerges from the cloud.

The 2-D simulation results exhibit strong unsteady effects. Shock reflections from a finite number of discrete particles create a noisy source of compression waves inside the particle cloud, long after the shock wave has left the cloud. Localized regions of transonic Mach numbers near the trailing edge of the cloud contribute to this source of compression waves. Additionally, the unsteady flow behind the cloud is characterized by vortical structures that propagate downstream. The contact discontinuity marks the interface between the unsteady flow region behind the particle cloud and the higher density fluid located just behind the transmitted shock. Similar flow features have been observed in the experimental work in Ref. 20.

An equivalent one-dimensional model problem is created using volume-averaged equations. The 1-D model neglects all unclosed fluctuation terms, such as the Reynolds stress term, and models the particle drag with the steady-state drag law and the undisturbed flow force. A drag coefficient is obtained that provides a match of the reflected and transmitted shock wave's locations and magnitudes. The 1-D model captures the one-dimensional flow behavior well, including the reflected and transmitted shock waves, the contact discontinuity, and the expansion across the cloud.

The 1-D model does not capture unsteady effects such as a time lag in the drag coefficient due to a delay in boundary layer separation, compression wave generation from localized regions where transonic flow conditions exist, and energy contained in unclosed fluctuation terms such as the Reynolds stress and turbulent kinetic energy. While it may be satisfactory to neglect these unclosed fluctuation terms in dilute flows, these terms play a dominant role in dense flows and must be modeled.

\section{Acknowledgments}

The authors would like to thank Karen Oren for her contribution to this work.

\section{References}

\footnotetext{
${ }^{1}$ Crowe, C. T., Schwarzkopf, J. D., Sommerfeld, M., and Tsuji, Y., Multiphase Flows with Droplets and Particles, CRC Press, 2nd ed., 2012.

${ }^{2}$ Zhang, F., Frost, D. L., Thibault, P. A., and Murray, S. B., "Explosive Dispersal of Solid Particles," Shock Waves, Vol. 10, 2001, pp. 431-443.

${ }^{3}$ Igra, O. and Ben-Dor, G., "Dusty Shock Waves," Applied Mechanics, Vol. 41, 1988, pp. 379-437.

${ }^{4}$ Ergun, S., "Fluid flow through packed columns," Chem. Engr. Prog., Vol. 48, 1952, pp. 89.

${ }^{5}$ Gibilaro, L. G., Di Felice, R., Waldram, S. P., and Foscolo, P. U., "Generalized Friction Factor and Drag Coefficient Correlations for Fluid-Particle Interactions," Chemical Engineering Science, Vol. 40, No. 10, 1985, pp. $1817-1823$.

${ }^{6}$ Rogue, X., Rodriguez, G., Haas, J., and Saurel, R., "Experimental and numerical investigation of the shock-induced fluidization of a particles bed," Shock Waves, Vol. 8, No. 1, Feb. 1998, pp. 29-45.

${ }^{7}$ Di Felice, R., "The Voidage Function for Fluid-Particle Interaction Systems," Int. J. Multiphase Flow, Vol. 20, 1994, pp. $153-159$.

${ }^{8}$ Carrier, G., "Shock waves in a dusty gas," J. Fluid Mech., , No. March, 1958.
} 
${ }^{9}$ Miura, H. and Glass, I. I., "On a Dusty-Gas Shock Tube," Proceedings of the Royal Society A: Mathematical, Physical and Engineering Sciences, Vol. 382, No. 1783, Aug. 1982, pp. 373-388.

${ }^{10}$ Miura, H. and Glass, I. I., "On the Passage of a Shock Wave Through a Dusty-Gas Layer," Proceedings of the Royal Society A: Mathematical, Physical and Engineering Sciences, Vol. 385, No. 1788, Jan. 1983, pp. 85-105.

${ }^{11}$ Miura, H., "Decay of shock waves in a dusty-gas shock tube," Fluid Dynamics Research, Vol. 6, No. 5-6, Dec. 1990, pp. 251-259.

${ }^{12}$ Saito, T., "Numerical Analysis of Dusty-Gas Flows," J Comp Phys, Vol. 176, No. 1, 2002, pp. 129-144.

${ }^{13}$ Baer, M. R. and Nunziato, J. W., "A Two-Phase Mixture Theory for the Deflagration-to-Detonation Transition (DDT) in Reactive Granular Materials," Int. J. Multiphase Flow, Vol. 12, No. 6, 1986, pp. 861-889.

${ }^{14}$ Powers, J. M., Stewart, D. S., and Krier, H., "Theory of Two-Phase Detonation-Part I: Modeling," Combustion and Flame, Vol. 80, No. 3-4, 1990, pp. 264-279.

${ }^{15}$ Baer, M. R., "Shock Wave Structure in Heterogeneous Reactive Media," 21st Int. Symp. Shock Waves, 1997 , pp. 923-927.

${ }^{16}$ Anderson, M. U., Graham, R. a., and Holman, G. T., "Time-resolved shock compression of porous rutile: Wave dispersion in porous solids," AIP Conference Proceedings, Vol. 309, No. 1994, 1994, pp. 1111-1114.

${ }^{17}$ Sommerfeld, M., "The unsteadiness of shock waves propagating through gas-particle mixtures," Experiments in Fluids, Vol. 3, No. 4, 1985, pp. 197-206.

${ }^{18}$ Boiko, V., Kiselev, V., Kiselev, S., a.N. Papyrin, Poplavsky, S., and Fomin, V., "Shock wave interaction with a cloud of particles," Shock Waves, Vol. 7, No. 5, Oct. 1997, pp. 275-285.

${ }^{19}$ Wagner, J. L., Beresh, S. J., Kearney, S. P., Trott, W. M., Castaneda, J. N., Pruett, B. O. M., and Baer, M. R., "Interaction of a Planar Shock with a Dense Field of Particles in a Multiphase Shock Tube," Proc. 49th AIAA Aerospace Sciences Meeting, Vol. 188, 2011, pp. 1-13.

${ }^{20}$ Wagner, J. L., Beresh, S. J., Kearney, S. P., Trott, W. M., Castaneda, J. N., Pruett, B. O., and Baer, M. R., "A multiphase shock tube for shock wave interactions with dense particle fields," Experiments in Fluids, Vol. (in press), Feb. 2012.

${ }^{21}$ Boiko, V. M. and Poplavski, S. V., "On the Dynamics of Drop Acceleration at the Early Stage of Velocity Relaxation in a Shock Wave," Comb. Exp. Shock Waves, Vol. 45, No. 2, 2009, pp. 198-204.

${ }^{22}$ Clemins, A., "Representation of two-phase flows by volume averaging," International Journal of Multiphase Flow, Vol. 14, No. 1, Jan. 1988, pp. 81-90.

${ }^{23}$ Roe, P. L. and Pike, J., "Efficient construction and utilisation of approximate Riemann solutions," Computing methods in applied sciences and engineering, VI, 1984, pp. 499-518.

${ }^{24}$ Toro, E. F., Riemann Solvers and Numerical Methods for Fluid Dynamics, Spring, 2nd ed., 1999.

${ }^{25}$ van Leer, B., "Towards the ultimate conservative difference scheme. V. A second-order sequel to Godunov's method," Journal of Computational Physics, Vol. 32, No. 1, 1979, pp. 101-136.

${ }^{26}$ Sun, M., Saito, T., Takayama, K., and Tanno, H., "Unsteady drag on a sphere by shock wave loading," Shock Waves, Vol. 14, No. 1-2, June 2005, pp. 3-9.

${ }^{27}$ White, F. M., Fluid Mechanics, McGraw Hill, New York, seventh ed., 2011.

${ }^{28}$ Regele, J. D. and Vasilyev, O. V., "An adaptive wavelet-collocation method for shock computations," International Journal of Computational Fluid Dynamics, Vol. 23, No. 7, Aug. 2009, pp. 503-518.

${ }^{29}$ Makkawi, Y., "The voidage function and effective drag force for fluidized beds," Chemical Engineering Science, Vol. 58, No. 10, May 2003, pp. 2035-2051.

${ }^{30}$ Saito, T., Saba, M., Sun, M., and Takayama, K., "The effect of an unsteady drag force on the structure of a nonequilibrium region behind a shock wave in a gas-particle mixture," Shock Waves, Vol. 17, No. 4, Oct. 2007, pp. $255-262$.

${ }^{31}$ Drew, D. A. and Passman, S. L., "Theory of Multicomponent Fluids," Applied Mathematical Sciences, Vol. $135,1999$. 